\title{
ANÁLISIS DE LA PRODUCTIVIDAD MEDIANTE REDES BAYESIANAS EN UNA PYME DESARROLLADORA DE TECNOLOGÍA Griselda Dávila-Aragón*
}

Universidad Panamericana, Escuela de Ciencias Económicas y Empresariales Fernando Cruz-Aranda

Universidad Panamericana, Escuela de Ciencias Económicas y Empresariales Agustín I. Cabrera-Llanos

Instituto Politécnico Nacional, Unidad Profesional Interdisciplinaria de Biotecnología Francisco Ortiz-Arango

Universidad Panamericana, Escuela de Ciencias Económicas y Empresariales

(Recibido 17 de septiembre 2014, aceptado 10 de diciembre 2014)

\begin{abstract}
Resumen
Las redes bayesianas (RB) son una herramienta de novedosa aplicación a la gestión de riesgos, en particular para modelar el riesgo operacional. Su uso para medir el riesgo operativo en el sector financiero ha encauzado grandes esfuerzos en desarrollar nuevos métodos de medir este tipo de riesgo que permitan mejorar la gestión interna de los procesos operativos. El aplicar redes bayesianas para modelar el riesgo operacional presenta la oportunidad de incorporar elementos cualitativos como la opinión de expertos en el proceso de seleccionar las variables de interés, definir la estructura del modelo por medio de sus dependencias de causalidad, así como la especificación de las distribuciones a priori y las probabilidades condicionales de cada nodo El objetivo del presente trabajo es aprovechar esta herramienta para poder llevar a cabo el análisis de la productividad de una PYME dedicada al desarrollo de tecnología.
\end{abstract}

\begin{abstract}
Bayesian Networks (RB) is a tool for risk management, particularly for modeling operational risk. Its use for measuring operational risk in the financial sector has channeled great efforts to develop new methods to measure this type of risk to improve the internal management of business processes. Applying Bayesian Networks to model operational risk has the opportunity to incorporate qualitative elements as the opinion of experts in the process of selecting the variables of interest, define the model structure through its causal dependencies and specifying the priors and conditional probabilities of each node the aim of this work is to use this tool to perform the analysis of productivity of PYMES dedicated to developing technology.
\end{abstract}

Clasificación JEL:C02, C11, C16, G29.

Palabras clave: Redes Bayesianas, Riesgo operacional, Tecnología.

\footnotetext{
* Plan de Agua Prieta 66, Del. Plutarco Elías Calles, C.P: 11350. México D. F Tel. 7717227110, (55) 57296000 Ext.62037. Correo electrónico: hriosb@hotmail.com
} 


\section{Introducción}

Las micro, pequeñas y medianas empresas (MYPIME) constituyen el mayor porcentaje de empresas en todos los países de América Latina y el Caribe, llegando a ser más del $90 \%$ del total de ellas. Sin embargo aunque participan entre el $25 \%$ y $45 \%$ de los empleos y entre el 15 y $25 \%$ de la producción, su productividad esta entre un 25 y $50 \%$, frente a las PYMES Europeas, cuya productividad está entre un 66 y $82 \%$ (Vergara, 2005).

Uno de los problemas más comunes en el medio empresarial micro y pequeño en México es la falta de cultura de medición. Un hecho innegable es que, para poder mejorar, es necesario controlar, y para poder controlar es indispensable medir. Todo en esta vida es susceptible de medición. Un mal silencioso en este sector es la falta de indicadores de desempeño, un tema que al no ser conocido puede parecer innecesario, sin embargo, los indicadores de productividad son una de las herramientas más valiosas para poder monitorear los procesos y plantear metas de desempeño en el corto y mediano plazo, la medición del desempeño es indudablemente el cimiento de la mejora de procesos ya que permite conocer los niveles de productividad y compararlos con periodos anteriores para poder observar un real incremento o decremento en el desempeño.

La productividad evalúa la capacidad de un sistema para elaborar los productos que son requeridos y a la vez el grado en que se aprovechan los recursos utilizados. Una mayor productividad utilizando los mismos recursos resulta en una mayor rentabilidad para la empresa, es por ello que toda empresa trata de aumentar al máximo su productividad.

En la empresa bajo análisis, como en la mayoría de las PYMES, no se tienen registros formales de la mayoría de las variables definidas, lo que en un principio dificulta la labor de investigación, sin embargo la metodología de Redes Bayesianas permite trabajar con la opinión de los expertos en la definición de dichas variables y sus probabilidades de ocurrencia en el caso de insuficiencia de datos estadísticos.

El presente trabajo es un caso aplicado a una empresa mediana dedicada al desarrollo tecnológico en la Ciudad de México, la cual, a pesar de su crecimiento en el mercado no tiene un sistema de indicadores de productividad, por lo que se desarrolló en conjunto con los administradores de la misma una metodología empleando Redes Bayesianas que le permita medir sus productividad en función de sus variables de riesgo y poder mejorar el desempeño de la organización.

El presente trabajo está organizado de la siguiente manera: En la sección 2 se plantea una breve descripción de las Redes Bayesianas y cómo se construye un modelo basado en ellas, posteriormente en la sección 3 se construye el modelo para medir el desempeño de la empresa, iniciando por identificar las variables, nodos e interacciones existentes a partir de la opinión de expertos, para concluir esta sección con la construcción de la red bayesiana del modelo y los cálculos del modelo en distintos escenarios obteniendo distintos resultados. Finalmente se presentan las conclusiones y bibliografía consultada. 


\section{Redes Bayesianas}

Las Redes Bayesianas son un tipo de modelo causal resultado de la convergencia de la evolución de la modelación estadística, ingeniería e inteligencia artificial que inició en la década de los 80s. (Alexander, 2002). Son una clase de modelo gráfico que puede ser usado para modelar dependencias causales entre las variables aleatorias para la determinación de factores de riesgo operacional y asignar el capital de riesgo operacional en empresas del sector financiero (Neil, Fenton y Tailor, 2005). La ventaja principal de esta clase de modelos es que permite estar en posibilidades de determinar las diferentes relaciones causales; las RB surgen como una herramienta para integrar criterios y aspectos cualitativos subjetivos con datos cuantitativos internos y externos facilitando la comprensión causal dentro de las estimaciones.

Una red bayesiana es una estructura matemática que se utiliza para representar el entendimiento de un proceso y sus dependencias (causa-efecto), asignándoles ponderaciones y probabilidades de ocurrencia. El problema al calcular las probabilidades numéricas es esencialmente el mismo que el que se tiene al realizar una autoevaluación de control de riesgo operacional. Es un grafo dirigido acíclico; los nodos representan las variables de interés y las aristas son los enlaces causales o de influencia entre las variables (Madsen, Kjaerulff 2008). A cada nodo se le asocia una tabla de probabilidad del nodo, así como una distribución estadística o una función parametrizada. En el caso de una tabla de probabilidad del nodo la relación se rige por un conjunto de valores de probabilidad condicionales que modelan la relación incierta entre el nodo y sus nodos padre junto con cualquier incertidumbre presente en esa relación.

La teoría detrás de las redes bayesianas combina la teoría de probabilidad bayesiana y la noción de independencia condicional para representar dependencias entre las variables.

Las redes bayesianas permiten razonar bajo incertidumbre y combinar las ventajas de la representación visual intuitiva con una base matemática de probabilidad bayesiana. Es posible articular dependencias entre diferentes variables y propagar sistemáticamente el impacto de la evidencia sobre las probabilidades de resultados inciertos. La representación gráfica de las redes bayesianas facilita el entendimiento de la estructura causal y el perfil de riesgo de la empresa se presenta de forma intuitiva (Neil, Fenton y Márquez, 2004).

La clave para un diseño exitoso de una red bayesiana es la descomposición significativa de un dominio del problema en un conjunto de proposiciones causales o condicionales sobre el dominio. En lugar de llevar a cabo la laboriosa y difícil tarea de calcular la distribución de probabilidad conjunta de todas las variables de interés, se aplica el principio "divide y vencerás" y tomar las especificaciones parciales del modelo que son en sí significativos en el dominio del experto (Chonawee 2006).

Posteriormente se modela la tabla de probabilidad del nodo para cada variable (nodo), lo cual puede ser hecho usando datos históricos o solamente pidiéndole a un experto que nos proporcione una serie de estimaciones subjetivas, las cuales estarán idealmente basadas en el conocimiento y la experiencia (Cowell 1999). 
Un experto es una persona cuya experiencia y conocimiento de la operación le permite hacer conjeturas suficientemente creíbles acerca de cómo las operaciones de la empresa afectan el perfil de riesgo de la misma. En un inicio su opinión puede ser utilizada como un sustituto de los datos y puede proporcionar información valiosa de las operaciones de la empresa que es difícil de capturar de los datos por sí solos. El reto del modelador es incorporar estas aportaciones en el modelo de medición. Una vez construida la red bayesiana, puede ser ejecutada usando un algoritmo apropiado de propagación. Cuando la red bayesiana se ejecuta, el efecto de los datos ingresados dentro de uno o más nodos son propagados por toda la red, en cualquier dirección y la distribución marginal de los nodos es actualizada. Esto hace al modelo ideal para un análisis de escenarios.

La oportunidad de incorporar la opinión de expertos en el proceso de seleccionar las variables de interés, definir la estructura del modelo por medio de sus dependencias de causalidad y la especificación de las distribuciones a priori y por consiguiente las probabilidades condicionales de cada nodo es una aplicación de un modelo de Redes Bayesianas a la medición del Riesgo Operacional de una empresa. Como se estableció antes en este trabajo se aplicó la teoría de Redes Bayesianas para medir la productividad de una PYME dedicada al desarrollo tecnológico. Dado su giro se tiene un gran capital invertido en software y hardware que soporten la operación. El objetivo del análisis es identificar la causalidad entre las variables que ocasionan baja productividad.

3. Desarrollo del modelo basado en redes bayesianas para medir la de la empresa

\subsection{Construcción del modelo.}

Consultando a los administradores de la empresa se definieron las siguientes variables para el diseño del modelo.

Tabla 1. Definición de las variables (nodos).

\begin{tabular}{|c|l|l|}
\hline & Variable & Valores \\
\hline 1. & Capital Humano & Calificado / No calificado \\
\hline 2. & Regulación & Desalentar / Incentivar \\
\hline 3. & $\begin{array}{l}\text { Inversión en Departamento } \\
\text { de Investigación }\end{array}$ & Propia / Gubernamental \\
\hline 4. & Seguridad Informática & Alta / Baja \\
\hline 5. & Rotación del personal & Alta / Baja \\
\hline 6. & Propiedad Intelectual & $\begin{array}{l}\text { Con Registro / Sin } \\
\text { Registro }\end{array}$ \\
\hline 7. & Soporte Tecnológico & Bueno / Regular / Malo \\
\hline 8. & $\begin{array}{l}\text { Innovación de Productos } \\
\text { Tecnológicos }\end{array}$ & $\mathrm{Si}$ / No \\
\hline 9. & Robo o Fraude & $\mathrm{Si} /$ No \\
\hline 10. & Productividad & $\mathrm{Alta} /$ Mediana / Baja \\
\hline
\end{tabular}




\subsection{Modelo Gráfico de las relaciones de causalidad}

Figura 1. Modelo gráfico de las relaciones de causalidad.

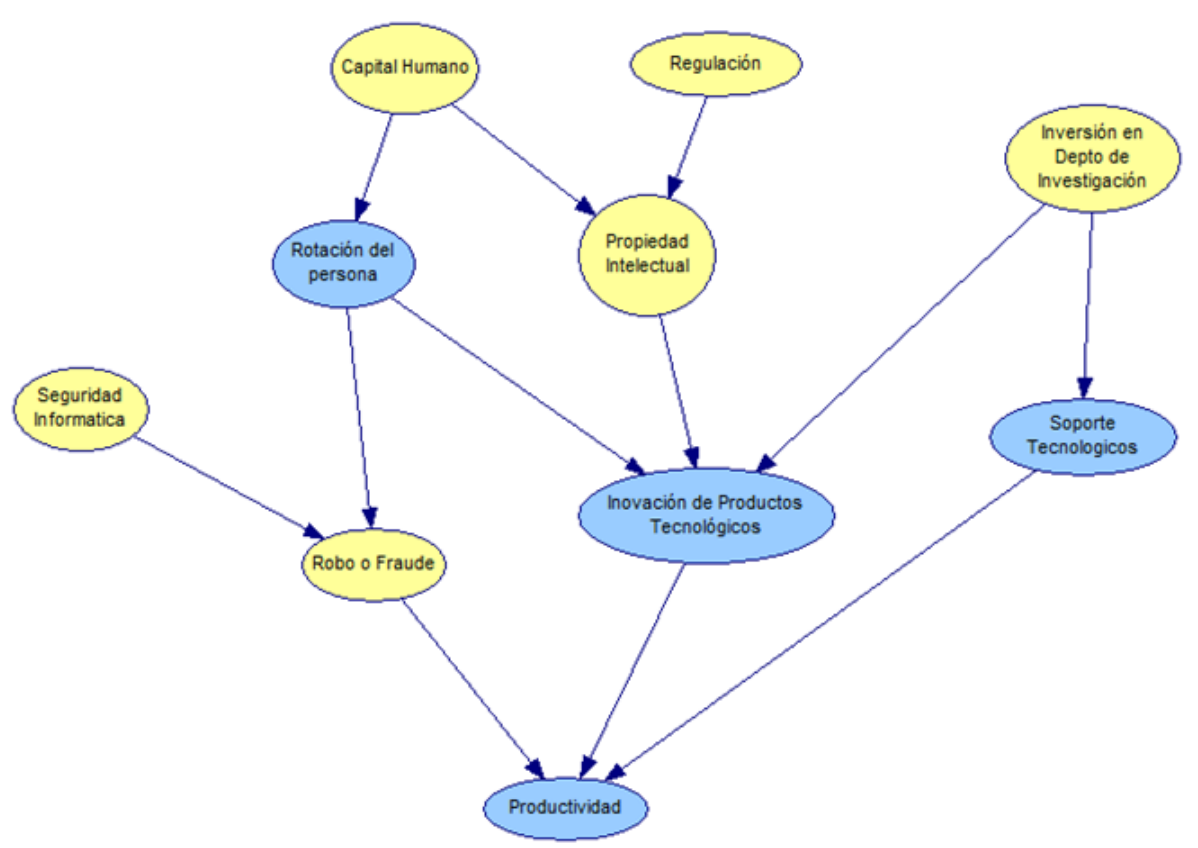

Una vez definido el modelo gráfico con el que se va a trabajar, es factible hacer inferencia a partir de él acerca de las distribuciones a posteriori. A continuación se procede a realizar los cálculos de la difusión de las interacciones entre los distintos tipos de nodos, sus ponderaciones así como las probabilidades asociadas, este proceso es bastante laborioso si se realiza de forma manual, afortunadamente existe software disponible en el mercado ya sea en versión gratuita o versiones comerciales para realizar las fases, para el presente trabajo se utilizó una versión libre del software GeNIe, lo cual que permite la obtención de las siguientes distribuciones: 


\subsection{Distribuciones marginales a priori}

Figura 2. Esquema presentado por software GeNIe, donde se presentan los nodos, interacciones y probabilidades asociadas.

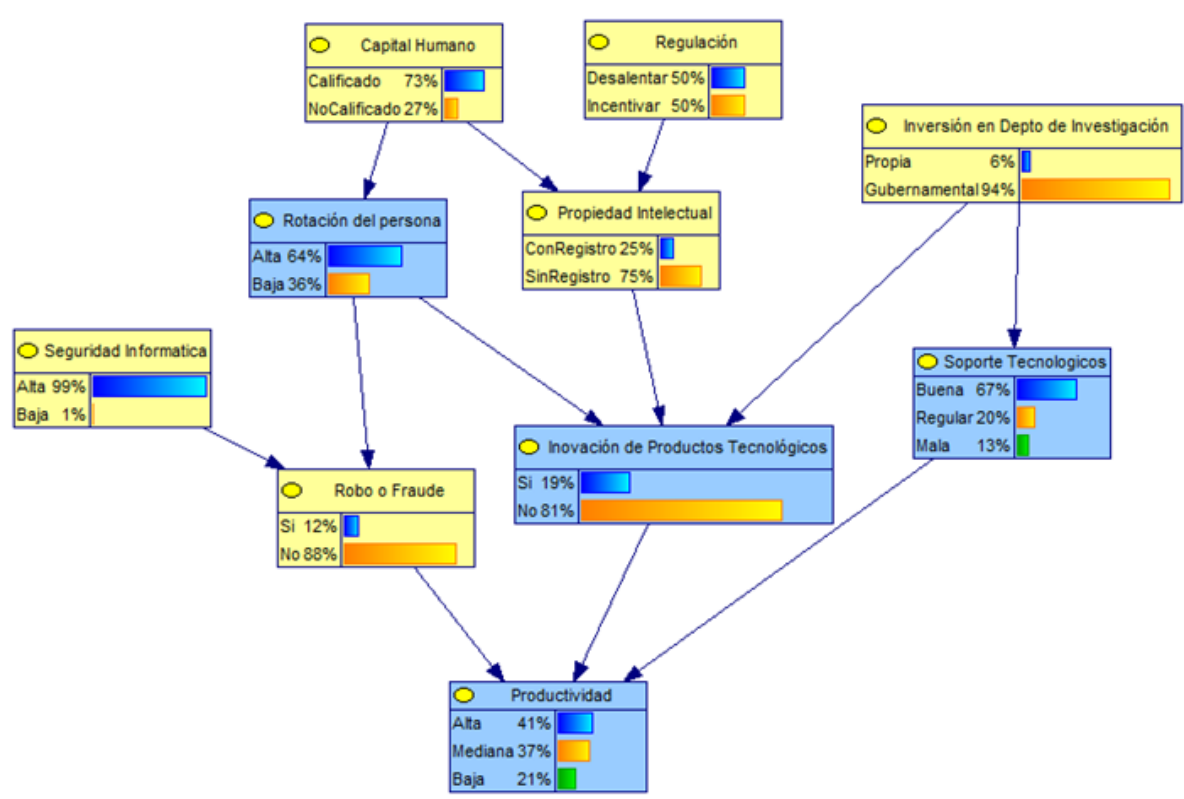

Una vez que se han alimentado los nodos, interacciones y probabilidades a priori en el sistema, es posible iniciar el proceso de cálculo. La Red Bayesiana está lista para modelar la productividad; es decir, el indicador de eficiencia que relaciona la cantidad de recursos utilizados con la cantidad de producción obtenida.

El modelo se alimenta con datos conocidos (históricos o provenientes de expertos) para determinar el estado real de la empresa. Se inicia sabiendo que la empresa no cuenta con apoyo gubernamental para inversión en el departamento de investigación, que tiene una excelente calidad de soporte tecnológico y todo su personal está altamente calificado y certificado en el desarrollo de productos tecnológicos; con estos datos el modelo se alimenta en la fase de inicialización con la evidencia proporcionada y se reinicia el árbol para obtener las nuevas distribuciones marginales a posteriori.

Los resultados obtenidos se muestran en la figura 3, en la cual se presentan las distribuciones marginales a posteriori las cuales se obtienes a partir de la evidencia o datos iniciales proporcionados por la empresa. 


\subsubsection{Distribuciones marginales posteriori basadas en la evidencia de la empresa.}

Figura 2. Esquema presentado por software GeNIe, donde se presentan los nodos, interacciones y probabilidades asociadas.

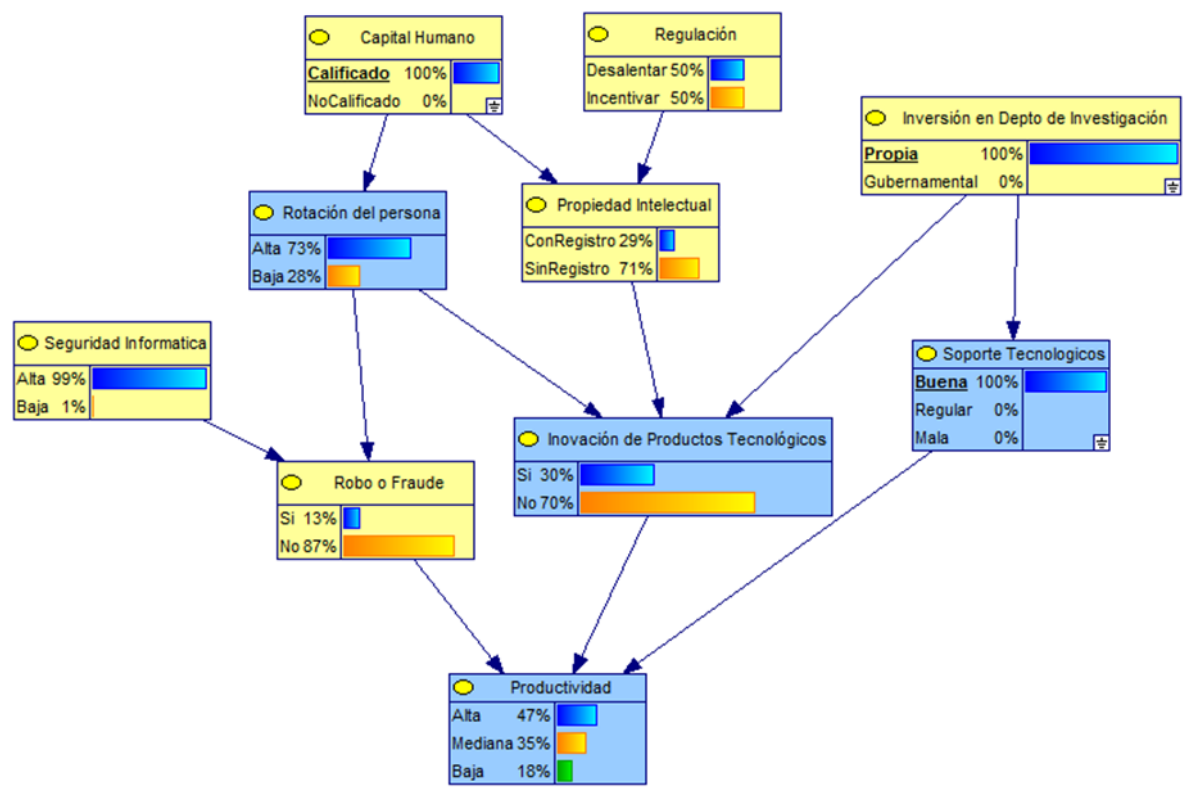

Los resultados obtenidos nos muestran que la productividad de la empresa es alta con una probabilidad del $47 \%$ y baja con probabilidad del $18 \%$. Una de las grandes ventajas de tener esta estructura y el uso del software, es que podemos realizar los cálculos de nuevos escenarios de manera rápida, así por ejemplo si la empresa tomara la decisión de disminuir costos dejando de registrar toda su propiedad intelectual; la decisión se incorpora al árbol y se propaga nuevamente para obtener las densidades marginales siguientes, a las cuales llamaremos Distribuciones marginales a posteriori con pruebas de escenario, la cuales podremos ver en la figura 4. 


\subsubsection{Distribuciones marginales posteriori con pruebas de escenario.}

Figura 4. Distribuciones marginales posteriori con pruebas de escenario

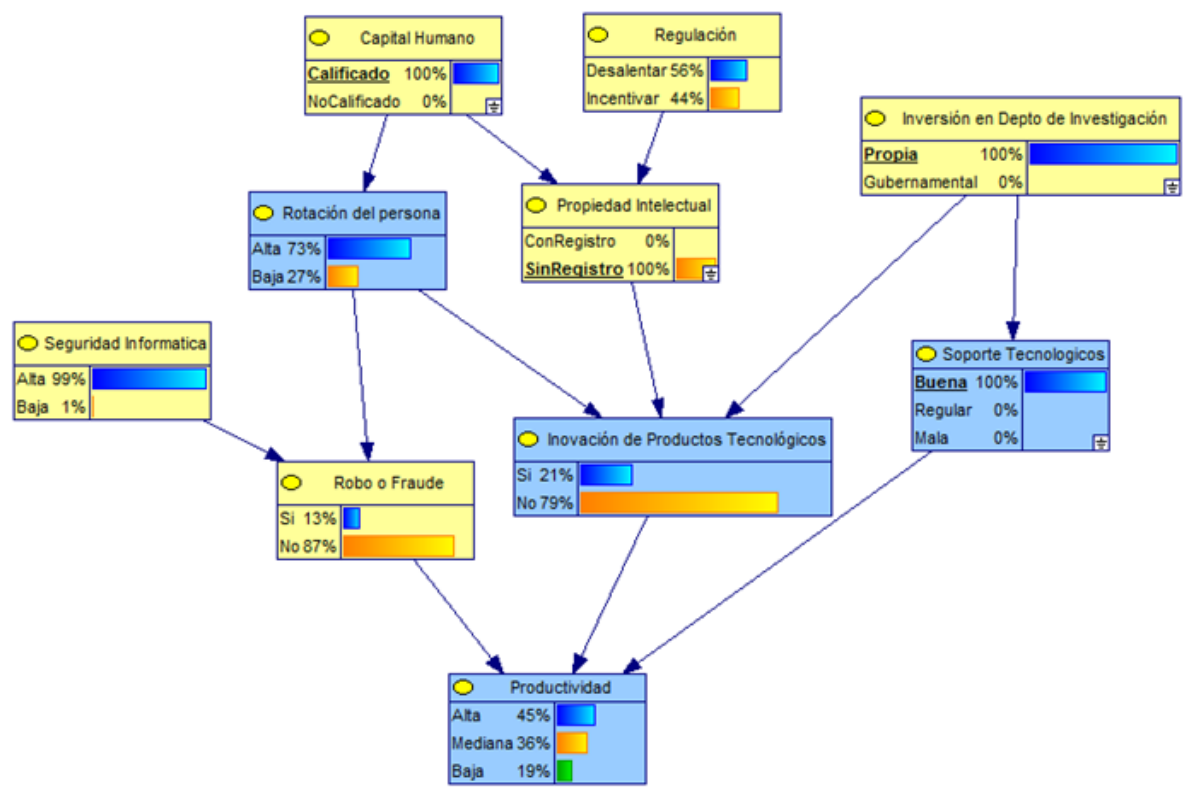

En este caso lo que podemos interpretar de estos nuevos resultados es que el ahorro repercute en una productividad alta con probabilidad del $45 \%$, mediana con probabilidad del $36 \%$ y baja con probabilidad del 19\%, probabilidades menores a las obtenidas anteriormente. Una gran ventaja del uso de Modelos de Redes Bayesianas es que permiten investigar el impacto de un evento adverso que se presente en la empresa, por medio de pruebas de estrés.Consideremos el caso de que la empresa fuera víctima de robo o fraude, cuyos resultados se muestran en la figura 5 . 


\subsubsection{Distribuciones marginales posteriori modelando causa-efecto: Robo o Fraude}

Figura 5.1

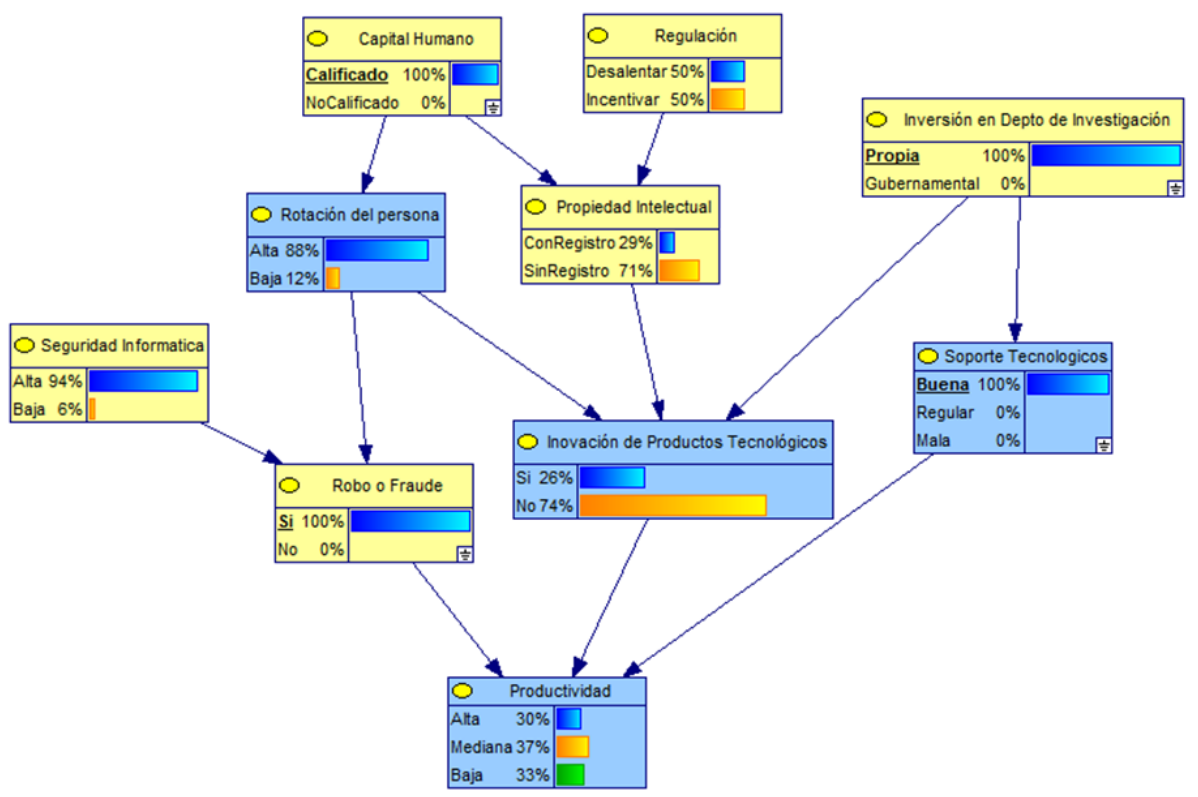

El efecto de este evento es una disminución en su probabilidad de una productividad alta, al disminuir a niveles del $30 \%$ y un aumento al $33 \%$ de obtener una baja productividad. En el caso de modificaciones a la regulación que desalentara el desarrollo de propiedad intelectual, los resultados serían los siguientes. 


\subsubsection{Distribuciones marginales posteriori modelando causa-efecto: Regulación desalentadora}

Figura 5. Distribuciones marginales posteriori modelando causa-efecto: Robo o Fraude

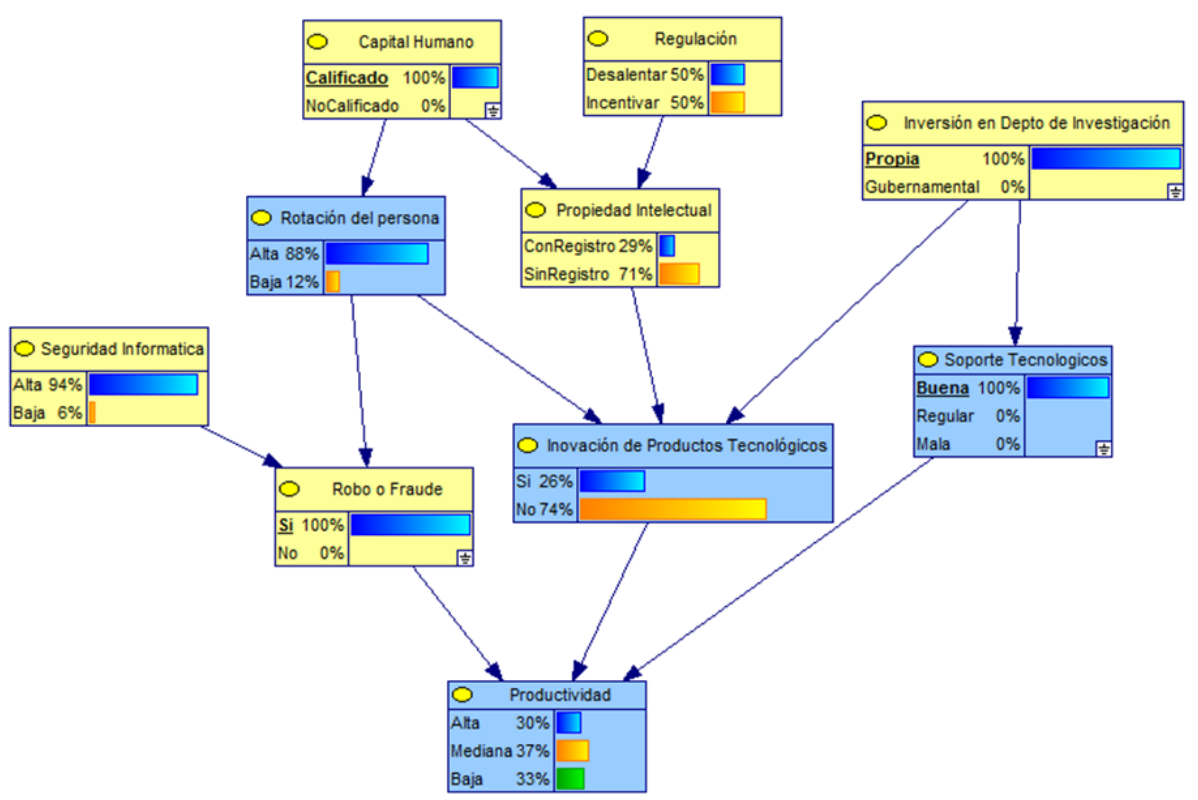

En este caso la probabilidad de registro de la propiedad intelectual disminuye de $29 \%$ a $21 \%$, la innovación en productos tecnológicos también disminuye de $30 \%$ a $27 \%$; sin embargo las probabilidades de productividad se mantienen contantes. De este modo cada nueva información obtenida respecto a las variables incluidas en el árbol puede y debe ser incorporada a la Red Bayesiana para mantener al modelo dinámico.

\section{Conclusiones}

El uso de Redes Bayesianas para modelar la productividad en la empresa incorpora la opinión de expertos mediante la selección de las variables de interés, la definición de la estructura del modelo por medio de las relaciones de dependencia y la especificación de las distribuciones a priori y las probabilidades condicionales de cada nodo.

La actualización de la red con la nueva información disponible permite garantizar que esta no es estática y el modelo no pierde valides con el tiempo. Las redes bayesianas proveen de un modelo dinámico por lo que la red se adapta rápidamente a la nueva información, incorporando la opinión de los expertos de forma matemática.

Las pruebas de estrés pueden ser fácilmente desarrolladas en las redes bayesianas, lo que las vuelve un sistema de alertas tempranas para el monitoreo de movimientos adversos en las variables de riesgo del modelo. El modelo 
puede volverse complejo si existen muchos nodos por especificar, especialmente si los nodos provienen de muchos padres. En estos casos, existirán varias probabilidades condicionales que definir que requerirán de un gran volumen de información; se podría utilizar el método de máxima verosimilitud para especificarlas, lo cual reduce una de las principales ventajas del uso de las redes bayesianas.

Uno de los mayores retos de las redes bayesianas es lidiar con la opinión de los expertos que por lo general no están familiarizados con términos estadísticos. Existe también la problemática de no tener una estructura de relación causal única del modelo, por lo que escoger el modelo que mejor ajuste se vuelve subjetivo y por consiguiente es sujeto a debate.

\section{Bibliografía}

Alexander, Carol (2002). Bayesian Methods for Measuring Operational Risk. Discussion Papers in Finance Circular nica de Bancos (CUB), Secretaría de Hacienda y Crédito Público, 2005.

Basel Committee on Banking Supervision (BCBS). 2006. International Convergence of Capital Measurement and Capital Standards, Bank for International Settlements.

Chonawee S. Chris K. And Lucas H. (2006). Cause to Effect Operational Risk Quantification and Management, Risk Management.

Cowell, R. G., A. P. Dawid, S. L. LAuritzen y D. J. Spiegelhalter, (1999). Probabilistic Networks and expert Systems, Springer-Verlag

Madsen, A. and Kjaerulff U. 2008. Bayesian Networks and Influence Diagrams. Springer,

Neil, M.N. Fenton and M. Tailor. (2005). Using Bayesian Networks to model expected and unexpected operational losses, Risk Analysis Journal.

Vergara, Sebastian. Pequeñas y Medianas Empresas en América Latina. División de Desarrollo Productivo y Empresarial, Comisión Económica para América Latina y el Caribe. CEPAL, 2005 